AperTO - Archivio Istituzionale Open Access dell'Università di Torino

Shared and task-specific muscle synergies of Nordic walking and conventional walking

This is a pre print version of the following article:

Original Citation:

Availability:

This version is available http://hdl.handle.net/2318/1650251

since $2017-10-25 T 14: 32: 05 Z$

Published version:

DOI:10.1111/sms.12992

Terms of use:

Open Access

Anyone can freely access the full text of works made available as "Open Access". Works made available under a Creative Commons license can be used according to the terms and conditions of said license. Use of all other works requires consent of the right holder (author or publisher) if not exempted from copyright protection by the applicable law. 


\section{IIIS AperTO}

UNIVERSITÀ

DEGLI STUDI

DI TORINO

This is the author's final version of the contribution published as:

Gennaro Boccia, Chiara Zoppirolli, Lorenzo Bortolan, Federico Schena, Barbara Pellegrini

Paper: Shared and task-specific muscle synergies of Nordic walking and conventional walking

SCANDINAVIAN JOURNAL OF MEDICINE AND SCIENCE IN SPORTS. None, 2017, pp: 1-14

DOI: $10.1111 / \mathrm{sms} .12992$

The publisher's version is available at:

https://doi.org/10.1111/sms.12992

When citing, please refer to the published version.

Link to this full text:

http://hdl.handle.net/2318/1650251 
DR GENNARO BOCCIA (Orcid ID : 0000-0001-8706-4098)

DR BARBARA PELLEGRINI (Orcid ID : 0000-0002-9626-2632)

Article type : Original Article

\section{Shared and task-specific muscle synergies of Nordic walking and conventional walking}

Gennaro Boccia $^{1,2}$, Chiara Zoppirolli ${ }^{1,3}$, Lorenzo Bortolan ${ }^{1,3}$, Federico Schena ${ }^{1,3}$, Barbara Pellegrini $^{1,3}$

${ }^{1}$ CeRiSM Research Centre for Sport, Mountain, and Health, University of Verona, Rovereto, (TN), Italy

${ }^{2}$ NeuroMuscularFunction Research Group, Department of Medical Sciences, School of Exercise and Sport Sciences, University of Turin, Turin, Italy.

${ }^{3}$ Department of Neuroscience, Biomedicine and Movement Science, University of Verona, Verona, Italy, University of Verona, Verona, Italy.

Corresponding author: Gennaro Boccia, PhD, NeuroMuscularFunction Research Group, School of Exercise and Sport Sciences, Department of Medical Sciences, University of Turin, Turin, Italy. E-mail: gennaro.boccia@unito.it - Telephone: +39 0117764756

Keywords: motor modules; motor primitives; muscle coordination; quadrupedal locomotion.

\section{ABSTRACT}

Nordic walking is a form of walking that includes a poling action, and therefore an additional subtask, with respect to conventional walking. The aim of this study was to assess whether Nordic walking required a task-specific muscle coordination with respect to conventional walking. We compared the electromyographic (EMG) activity of 15 upper and lower limb muscles of nine Nordic walking instructors, while executing Nordic walking and conventional walking at $1.3 \mathrm{~ms}^{-1}$ on a treadmill. Non-negative matrix factorization method was applied to identify muscle synergies, representing the spatial and temporal organization of muscle coordination. The number of muscle synergies was not different between Nordic This article has been accepted for publication and undergone full peer review but has not been through the copyediting, typesetting, pagination and proofreading process, which may lead to differences between this version and the Version of Record. Please cite this article as doi: $10.1111 /$ sms. 12992

This article is protected by copyright. All rights reserved. 
walking (5.2 \pm 0.4$)$ and conventional walking $(5.0 \pm 0.7, \mathrm{p}=0.423)$. Five muscle synergies accounted for $91.2 \pm 1.1 \%$ and $92.9 \pm 1.2 \%$ of total EMG variance in Nordic walking and conventional walking, respectively. Similarity and cross-reconstruction analyses showed that four muscle synergies, mainly involving lower limb and trunk muscles, are shared between Nordic walking and conventional walking. One synergy acting during upper limb propulsion is specific to Nordic walking, modifying the spatial organization and the magnitude of activation of upper limb muscles compared to conventional walking. The inclusion of the poling action when Nordic walking do not require an increased complexity of movement control, making Nordic walking suitable for adapted physical activity programs that involve also subjects with low motor skill.

\section{INTRODUCTION}

Nordic walking is a form of total body exercise obtained by adding the use of walking poles to conventional walking. Nordic walking has become a popular physical activity recommended not only for healthy adult $[1,2]$ but also for elderly people [3]. Moreover, it has been increasingly adopted as physical activity useful for overweight [4-6] and neurological patients [7, 8]. This success arises not only from the benefits gained by its practise, but also from the fact that it is relatively easy to perform. The Nordic Walking technique suggested by INWA (International Nordic Walking Association) guidelines, has been developed with the aim to maintain a natural, biomechanically-correct walking and an appropriate posture. Nordic walkers apply propulsive force through the poles, combining a pushing action of one pole with the contralateral lower-limb push-off, at each walking stride. Upper body is thus significantly engaged in the propulsion during Nordic walking, resulting in a higher use of upper body musculature [9-11] and a greater energy expenditure with respect to conventional walking at a given speed [6, 10, 12-14]. Arm movements can be considered as an additional task with respect to conventional walking, leading to hypothesise that this might increase movement complexity and coordinative demand. To date, no information is available about the coordination strategies employed in Nordic walking locomotion. Filling out this gap of knowledge should be very important, particularly in reference to the widespread use of Nordic walking with frail populations $[8,15,16]$.

This article is protected by copyright. All rights reserved. 
Evaluating muscle coordination in the framework of muscle synergies is a method that has the potential to give a global picture of muscle coordination strategies [17]. Muscle synergy could be defined as a synchronic activation of a group of muscles. For this reason, muscle synergies simplify the control of complex movements reducing the number of degrees of freedom that the nervous system might specify for motor control $[18,19]$. Thus, muscle synergies are considered to be the basic control signals responsible for generating the muscles activation needed for executing a specific motor task [20-22]. The most used algorithm to evaluate muscle synergies is called non-negative matrix factorization (NNMF) [23]. It models the activity of many recorded muscles as a linear combination of a small set of time-invariant muscle synergies, i.e. muscle-weighting, each activated by a time-varying activation coefficient [19]. Muscle-weighting is defined as the spatial feature that represents individual muscle contributions for recruiting muscle synergy, and the synergy activation indicates timevarying coefficients of the synergy recruitment $[23,24]$.

A small number of muscle synergies (usually four or five) has been demonstrated to describe the organization of muscle activation during human walking [25]. Importantly, these muscle synergies have been associated to the functional subtasks of gait, suggesting that synergistic control of muscles reflects biomechanical goals [26-29]. As synergies are associated to functional subtasks, the number of synergies provides information about the complexity of motor control $[30,31]$. Thus, one can expect that adding a propulsive upper body action to the conventional walking might increase the number of the functional subtasks, consequently increasing the number of muscle synergies. Moreover, comparing muscle synergies between conventional walking and Nordic walking allow the identification of similarities and/or differences in the motor control of their subtasks. There is a growing body of literature investigating the effect of speed, ground slope, and locomotion mode (i.e walking vs running) on muscle synergies involved in human locomotion. It is accepted that at least some muscle synergies are shared across a range of walking speeds [25, 32] and between walking and running [32], the muscle weightings of each synergy was found to be modulated to accomplish a wide repertoire of human locomotion conditions [33, 34]. Therefore, synergy analysis seems to be a suitable method to probe the difference in muscle coordination between conventional walking and Nordic walking.

While it is well established that Nordic walking brings an increment in the activation of upper body muscles with respect to conventional walking, it is not known whether this causes a change in the pattern of coordination. Thus, the aim of this study was to investigate the effect of Nordic walking on muscle coordination compared to conventional walking. As

This article is protected by copyright. All rights reserved. 
far as the poling action could be considered as an additional subtask, our hypothesis was that poling leads to an increase in the complexity of the locomotion, thus increasing the dimensionality of muscle synergies in Nordic walking compared to conventional walking. We also hypothesized that the two forms of locomotion only partially share the same muscle synergies, because the use of upper limbs in the propulsion introduces some changes in the coordination pattern.

\section{MATERIALS AND METHODS}

\section{Participants}

The study population was 9 (5 males, 4 females) Nordic walking instructors (mean age $39 \pm 12$ years, height $1.70 \pm 0.08 \mathrm{~m}$, body weight $62.8 \pm 8.2 \mathrm{~kg}$ ) licensed by the ANWI (Associazione Nordic Walking Italia) and with at least 2 years of experience in Nordic walking (mean $3 \pm 1$ years). The participants were recruited on a voluntary base, part of them were recruited directly by contacting instructors whose name was obtained by local offices of the Nordic Walking association (ANWI) and part of them were contacted as being acquaintances of the aforementioned participants. The general health status was normal; none had any health condition that could affect exercise capacity. Participants were instructed to refrain from performing strenuous physical activity in the $24 \mathrm{~h}$ before the experimental session. All the participants provided their written informed consent before participating in the experiments. The study was approved by the local Ethical Committee (Department of Neuroscience, Biomedicine and Movement Science, University of Verona) and performed in accordance with the Helsinki Declaration.

\section{Procedure}

Tests were performed on a motorized treadmill with a belt surface $2.5 \mathrm{~m}$ wide and 3.5 m long (RL3500E, Rodby, Sweden). Participants were requested to perform five minutes of either conventional walking or Nordic walking. EMG signals were recorded for $30 \mathrm{~s}$ during the last minute of exercise to ensure the acquisition of almost 20 cycles. Participants wore their habitual shoes and used NW poles (Exel, Nordic Walker, Espoo, Finland) equipped with special carbide tips to ensure appropriate grip with the treadmill belt surface. As recommended by the INWA, correct pole length was determined by multiplying the subject's height in $\mathrm{cm}$ by 0.68 rounded down to the nearest $5 \mathrm{~cm}$ within a tolerance of $2.5 \mathrm{~cm}$.

This article is protected by copyright. All rights reserved. 
An infrared reflective marker (diameter $1.4 \mathrm{~cm}$ ) was attached to the right heel to detect heel strike [35]. Heel position was recorded at a sampling rate of 100 samples/s using an optoelectronic motion capture system (6 cameras, MCU240, ProReflex; Qualisys, Gothenburg, Sweden). EMG signals were recorded at a sample rate of 2048 samples/s using multichannel amplifier (EMG-USB2, OT Bioelettronica, Turin, Italy) with a recording bandwidth 10-500 Hz. Bipolar Ag/AgCl surface EMG electrodes (Spes Medica, Battipaglia, Italy), with 2-cm inter-electrode distance, were placed over 15 muscles of the right side of the body including: Tibialis Anterior (TA), Soleus (SO), Gastrocnemius Medialis (GM), Vastus Lateralis (VL), Rectus Femoris (RF), Biceps Femoris (BF), Semitendinosus (ST), Gluteus Medius (Glu), Upper Trapezious (UT), Erector Spinae (ES) at the level of L2-L3, Latissimus Dorsi (LD), Anterior Deltoid (AD), Posterior Deltoid (PD), Biceps Brachii (BB), Triceps Brachii (TB). Before the placement of the electrodes, the skin was slightly abraded with abrasive paste and cleaned with water in accordance with SENIAM recommendations [36]. The optimal position and orientation of the electrodes were sought for each muscle following guidelines previously described [37]. A preliminary test was performed to check for cross talk and cable-induced noise and, when needed, electrodes and cables were repositioned. The electrodes and cables were fixed and secured on the body of the participants with an extensible dressing (Fixomull $^{\circledR}$, Beiersdorf, Hamburg, Germany) to avoid movement artifacts.

\section{EMG processing}

Data were analyzed by custom-written software in MATLAB R2015a (Mathworks, Natick, Massachusetts). Before processing, the EMG signals were carefully inspected, checking for noise and movement artifacts. For each trial, 10 to 25 consecutive, noncorrupted (i.e. without movement artefacts) gait cycles were selected for analysis [38]. Raw EMG signals were band-pass filtered (bi-directional, 4th-order, zero lag Butterworth, band-width $20-400 \mathrm{~Hz}$ ) to attenuate motion artefacts. The electrocardiogram contamination was removed from the EMG signals of trunk and upper limbs using the method proposed by Willigenburg and collegues [39]. All signals were then rectified and low-pass filtered at $9 \mathrm{~Hz}$ (bidirectional, 4th-order, zero lag Butterworth), resulting in the EMG envelopes [40].

This article is protected by copyright. All rights reserved. 
For both conventional walking and NW, the beginning of each cycle was considered from the local maximum forward position in the sagittal plane of the right heel marker [35]. The EMG envelopes were then re-sampled on a 100-point vector by means of a cubic spline interpolation. The EMG amplitude was normalized to the average of peak values across the available cycles of NW. Thus, EMG signals resulted into an $m \times t$ matrix $(\mathbf{E})$, where $m$ indicates the number of muscles (15) and $t$ is the time base (100 samples $\times$ number of gait cycles).

Differences across EMG patterns were assessed with the cross-correlation analysis $[41,42]$. This analysis provided two values: i) $r_{\max }$ coefficient (that is the maximum of the cross-correlation function) which gives an indication of the similarity of shape between EMG envelopes; ii) lag time, that is the magnitude of the shift between EMG envelopes.

\section{Muscle synergy analysis}

As done in previous studies [41-44], NNMF was performed from a set of consecutive gait cycles. The advantage of this technique is to take into account the inter-cycle variability $[38,45]$. For this purpose, we implemented the Lee and Seung algorithm [46, 47]. Matrix factorization minimizes the residual Frobenius norm between the initial matrix and its decomposition, given as follows:

$$
E=W C+e=E r+e
$$

Where $\mathbf{E}$ and $\mathbf{E}_{\mathbf{r}}$ are $m \times t$ matrix, $\mathbf{W}$ is a $m \times s$ matrix (where $s$ is the number of synergies), $\mathbf{C}$ is a $s \times t$ matrix, and $\mathbf{e}$ is a $m \times t$ matrix. $\mathbf{E}$ represents the initial EMG matrix, $\mathbf{E}_{\mathbf{r}}$ represents the reconstructed EMG, W represents the muscle synergy vector matrix, $\mathbf{C}$ represents the synergy activation coefficient matrix, and $\mathbf{e}$ is the residual error matrix, i.e., the difference between $\mathbf{E}$ and $\mathbf{E}_{\mathbf{r}}$, typically related to noise [48]. The algorithm is based on iterative updates of an initial random guess of $\mathbf{W}$ and $\mathbf{C}$ that converge to a local optimal matrix factorization (see Lee and Seung 2001 for more details).

To avoid local minima, the algorithm was repeated 40 times for each subject. The solution that minimized the squared error between original and reconstructed EMG patterns was kept. The number of synergies being an input of the NNMF algorithm, the algorithm was run from two to nine synergies. Then we selected the least number of synergy that accounted for $>90 \%$ of the variance accounted for $\left(\mathrm{VAF}_{\text {total }}\right)[41,44]$ while adding an additional synergy did not increase $\mathrm{VAF}_{\text {total }}$ by $>3 \%$. VAF was also computed for each muscle individually

This article is protected by copyright. All rights reserved. 
$\left(\mathrm{VAF}_{\text {muscle }}\right)$ to ensure that each muscle activity pattern was well accounted for the extracted muscle synergies, using a threshold of 75\% [41, 49]. To allow comparisons among individuals and motor tasks, muscle synergy vectors $(\mathbf{W})$ were normalized by the norm of each vector, as previously suggested $[50,51]$. The corresponding activation coefficients $(\mathbf{H})$ were scaled by the same quantity $[50,51]$.

As previously suggested [52], in an arbitrary subject the synergies were ordered according to the timing of the main peak of their activation coefficient. Then the synergies in the other subjects were sorted on the base of the values of synergy vectors similarity (see next paragraph) with that of the reference subject. This procedure assured the best matching between synergies across subjects and conditions [52].

To facilitate the comparison of the set of synergies between conditions and participants, the same number of muscle synergies, i.e. five, were extracted. This choice was acceptable since previous investigations, that analyzed a comparable number of muscles including those of upper limbs, showed that five muscle synergies well describe the pattern of muscle activation in conventional walking $[25,32,53]$.

\section{Assessment of similarity between muscle synergies.}

Similarity analysis between muscle synergies was done for each participant. It was conducted by computing the scalar product between pairs of vectors normalized by the product of their norms, which prioritizes the comparison between the shape of the vectors rather than amplitude [20]. Values above 0.80 has been used to define if a pair of vector is similar [27, 38]. The activation coefficients $\mathbf{C}$ were compared using cross-correlation analysis. We used two criteria: $r_{\max }$ (the maximum of the cross-correlation function) and lag time (the magnitude of the shift between synergy activation coefficients) [54, 55]. If the synergy vectors of the muscle synergies were similar between conventional walking and Nordic walking, we also examined the differences in the duration and magnitude of the activation patterns $[50,51]$. As previously recommended, the duration of the activity was defined as the full-width at half-maximum (FWHM) of the main peak $[32,52,56]$. The magnitude of the activity was examined by calculating the root mean square (RMS) of the activity during the FWHM [52]. Both the FWHM duration and RMS was calculated for each subject and each condition on the activation coefficients averaged across available cycles.

This article is protected by copyright. All rights reserved. 


\section{Cross-validation of the extracted synergy}

To verify the robustness of the extracted muscle synergies between the two tasks, we used a cross-validation procedure as proposed by previous researchers [21, 41, 45, 57-59]. For each participant, we checked whether the muscle synergy vectors $\mathbf{W}$ extracted from Nordic walking accounted for EMG envelopes of conventional walking. To do this, the muscle synergy matrix (i.e. muscle synergy vectors) extracted from Nordic walking was held fixed in the algorithm and the coefficients matrix $\mathbf{C}$ was free to vary with the multiple updating role until convergence [46]. The cross-reconstruction procedure was carried out separately for each subject. $\mathrm{VAF}_{\text {total }}$ and $\mathrm{VAF}_{\text {muscle }}$ values were calculated to evaluate the quality of this cross-reconstruction. We assumed that if conventional walking and Nordic walking were modulated by similar muscle synergies, the use of muscle weightings obtained from Nordic walking could be used for successfully reconstructing the EMG data of conventional walking and vice-versa $[60,61]$. A threshold of $80 \%$ for $\mathrm{VAF}_{\text {total }}$ reconstruction has been proposed as an indicator of acceptable reconstruction accuracy [55, 60-62]. However, to determine if the change induced by the task nature was higher than the withintask variability, we firstly determined the robustness of the cross-reconstruction within each task $[55,63]$. Thus, to determine the within-task variation of the synergies we firstly applied the cross-reconstruction analysis within each condition. For each condition, we divided the available duration of EMG signals into two parts: the first $30 \%$ (part 1) and the remaining $70 \%$ of time (part 2). The muscle synergy vectors extracted from part 1 were used to reconstruct the EMG pattern of part 2 of the same task. As previously suggested [55], we considered as a threshold of similarity the upper limit of the $95 \%$ confidence interval of the $\mathrm{VAF}_{\text {total }}$ change when EMG patterns of the part 2 were reconstructed using the synergy vectors of part 1. Thus, we considered that the synergies of conventional walking were significantly affected by NW if the $\mathrm{VAF}_{\text {total }}$ was reduced more than this threshold.

\section{Analysis on a subset of muscles}

To examine if differences in synergy composition between tasks were only caused by the intervention of upper limbs in Nordic walking we extracted muscle synergies on a subset of muscles (10 out of 15 muscles), removing the following five upper limb muscles: LD, AD, PD, BB, TB. Moreover, EMG of upper limb muscles in conventional walking usually presents a low signal-to-noise ratio [40] and this may influence the goodness of synergy extraction in conventional walking [23, 27, 32]. Upper limb muscles were mainly involved in the poling phase of Nordic walking and conversely were very low activated in the 
conventional walking. Since the modification of the number of muscles might affect the number of synergies extracted [64], we re-analysed the synergy dimensionality considering this subset of muscles. Furthermore, we reanalysed the quality of the cross-reconstruction using the subset of muscles.

\section{Statistical analysis}

Statistical analysis was performed in MATLAB R2015a (Mathworks, Natick, Massachusetts). Kolmogorov-Smirnov normality test was used to assess distributions normality. If the continuous data were not normally distributed were log-transformed before statistical analysis and back-transformed to obtain descriptive statistics. Significant differences in $\mathrm{VAF}_{\text {total }}, \mathrm{VAF}_{\text {muscle}}$, FWHM, and RMS between conventional walking and Nordic walking were calculated using paired $t$-tests. Student's paired $t$ tests were also used to compare the VAF values between the original and the cross-reconstructed EMG signals. Wilcoxon signed rank test was used to test differences between the number of synergies between conditions. To identify a shift in the time lag of EMG envelopes and muscle synergy coefficients, sample Student's $t$ tests with zero as reference value was performed.

\section{RESULTS}

\section{Individual EMG patterns}

The average ( $\pm 95 \%$ CI) EMG envelopes are reported in Figure 1. Table 1 shows the coefficients of similarity and lags between envelopes shapes. All muscle showed high similarity coefficients (all $r_{\max } \geq 0.85$ ). The higher indexes of similarity $\left(r_{\max } \geq 0.96\right)$ were found in the muscles of the lower-body (TA, SO, GM, VL), whereas the lower indexes $\left(0.85 \leq r_{\max } \leq 0.87\right)$ were found mainly in the muscles of the upper-body ( $\mathrm{LD}, \mathrm{AD}, \mathrm{BB}, \mathrm{TB}$, and $\mathrm{BF})$.

All muscles except LD, PD, TB were activated in the same gait phases in both conditions, indeed they did not show lags in the EMG envelopes between the two conditions (Table 1). In particular, TA, BF, and ST reached their peak of activation close to the instant of heel strike ( $0 \%$ of cycle); VL, RF, GLU, and ES (first peak) reached their peak at the $10 \%$ of gait cycle; SO and GM at the $40 \%$ of gait cycle (Figure 1). Differently, LD, PD, and TB, which are the muscles mainly involved in the poling action, showed their activity peak from $12 \%$ to $17 \%$ later in Nordic walking than in conventional walking (Table 1), thus resulting in an activity peak around the $60-70 \%$ of gait cycle in Nordic walking, compared to the $50 \%$ in conventional walking (Figure 1).

This article is protected by copyright. All rights reserved. 


\section{Dimensionality of muscle synergies}

Figure 2 shows the variance accounted for $\left(\mathrm{VAF}_{\text {total }}\right)$ each number of muscle synergies for each condition. Table 2 shows the individual values for the number of synergies extracted which was 4, 5, and 6 synergies in $11 \%, 66 \%$, and $22 \%$ of occasions, respectively. Contrary to our hypothesis, the number of muscles synergies did not change between the locomotion modes (conventional walking 5.0 \pm 0.7 , Nordic walking 5.2 $\pm 0.4, \mathrm{p}=0.423$ ). Analyzing individual results, three subjects (S4, S6, S7) showed an additional synergy, whereas one subject (S3) presented one less synergy in Nordic walking than in conventional walking (Table 2).

To compare the synergy compositions between tasks, we used five muscle synergies for all participants in both tasks (see Methods). The $\mathrm{VAF}_{\text {total }}$ explained by five synergies was greater in conventional walking $(92.9 \pm 1.2 \%)$ compared to Nordic walking $(91.2 \pm 1.1 \%$, $\mathrm{p}=0.002)$.

Figure 3 shows the $\mathrm{VAF}_{\text {muscle }}$ calculated when the selected five synergies were extracted. The $\mathrm{VAF}_{\text {muscle }}$ in conventional walking ranged from $76.6 \pm 12.2 \%$ (ES) to $96.1 \pm 1.6 \%$ (GM), in Nordic walking ranged from $81.3 \pm 8.5 \%$ (ES) to $95.4 \pm 2.2 \%$ (TB). Thus, on average all muscles were well represented by the five muscle synergies in both tasks. However, while in conventional walking the upper limb muscles (LD, PD, BB, TB) showed on average low values of $\mathrm{VAF}_{\text {muscle }}$ (ranging from $76.6 \pm 12.2 \%$ to $82.1 \pm 4.2 \%$ ), in Nordic walking these muscles showed consistently higher values (ranging from $89.1 \pm 6.2 \%$ to $95.4 \pm 2.2 \%)$.

\section{Functional characterization of muscle synergies}

Figure 4 shows the sets of muscle synergy vectors $(\mathbf{W})$ and the corresponding activation coefficients (C) for conventional walking (blue) and NW (red). In both conventional walking and Nordic walking, the five extracted muscle synergies were similar to those reported previously for walking $[25,32,38]$ and could be related to the subtasks of the gait cycle.

Synergy \#1 ("leg deceleration" function) mainly represented the activation of BF and ST (knee flexors/hip extensors) and TA (ankle dorsiflexor), being active during late swing and the initial contact phase.

Synergy \#2 ("weight acceptance" and "single leg support" functions): mainly represented the activation of VL and RF (knee extensors), and Glu (hip abductor) and was activated during the early stance.

This article is protected by copyright. All rights reserved. 
Synergy \#3 ("propulsion" function) mainly represented the activation triceps surae muscles, i.e. SO and GM (plantar flexors), and was activated in the late stance phase.

Synergy \#4 mainly represented the activation of ES (trunk extensor) and a spread activation of upper limb muscles. This synergy was activated mainly in the late stance and is related to "trunk extension" and "upper limb swing" functions.

Synergy \#5 mainly represented the activation of TA (ankle dorsiflexor) and upper limb muscles. This synergy was activated later than synergy \#4, during the leg swing phase. In conventional walking, this synergy is more related to "ankle dorsiflexion" and "upper limb swing" functions, since mainly represented the activity of TA (ankle dorsiflexor) and AD, BB, and UT (shoulder flexors and stabilizer, respectively). In Nordic walking, this synergy has the function of "upper limb propulsion", indeed the muscles LD, PD, and TB were pronouncedly activated as elbow and shoulder extensors.

\section{Comparison of muscle synergies}

The results of the similarity analyses are reported in Table 3. The normalized scalar product between the muscle synergy vectors (muscle weightings) were high for synergies \#1, $\# 2$, \#3, and \#4 ( $r \geq 0.81$, Table 3), indicating that the composition of these muscle synergies was similar across conventional walking and Nordic walking (spatial similarity). Regarding the activation coefficients, muscle synergies \#1, \#2, \#3, and \#4 can be considered robust across conditions and activated with the same profiles in the two tasks. Indeed, the shape $\left(r_{\max } \geq 0.91\right)$, the magnitude (all $p$ values $\geq 0.12$ ), and the duration (all $p$ values $\geq 0.38$ ) of the main peak for these synergies were similar in conventional walking compared to Nordic walking (Table 3).

Despite the overall similarity, some differences can be highlighted between tasks (Fig 2). For example, during Nordic walking the muscles around the hip are in general less represented in synergies \#1 (BF and ST) and \#2 (RF and Glu) compared to conventional walking. Moreover, the activation of upper limb muscles acting during the shoulder flexion (UT, $\mathrm{AD}$, and $\mathrm{BB}$ ) were spread differently across synergies: while these muscles are the most represented muscle in synergy \#5 of conventional walking, they showed higher weightings in the synergy \#1 of Nordic walking.

Synergy \#5 is of particular interest because consisted in the activation of upper limb muscles that were activated during the poling action of Nordic walking. It was the only synergy that showed significant differences with respect to conventional walking (normalized scalar product $=0.55)$. The main difference between the two tasks were that in Nordic 
walking the TA was less activated and the LD, PD, and TB were more activated than in conventional walking. Despite it was activated in the same gait phase and with the same duration in both tasks, synergy \#5 presented a trend to change in the shape of the activation coefficient $\left(r_{\max }=0.81\right)$. Overall these results suggest that in Nordic walking the synergy \#5 became clearly organized to produce the upper limb propulsion.

\section{Cross-validation of muscle synergies}

Table 2 shows the results of the cross-reconstruction analysis. When reconstructing EMG the pattern of conventional walking by using the synergy vectors obtained from Nordic walking, the cross-reconstruction showed significantly lower $\mathrm{VAF}_{\text {total }}$ values (original $92.9 \pm 1.2 \%$, cross-reconstructed $79.6 \pm 4.4 \%, \mathrm{p}<0.001)$. Despite the reasonable reconstruction $(\approx 80 \%)$ for the group considered as a whole, four subjects out of nine (S3, S4, S5, S6) did not reach the threshold of $80 \%$ for $\mathrm{VAF}_{\text {total }}$ (Table 2). Then we examined if the change in $\mathrm{VAF}_{\text {total }}$ due cross-reconstruction between tasks was greater than the change in $\mathrm{VAF}_{\text {total }}$ within conventional walking. We firstly calculated the upper limit of the $95 \%$ confidence interval of the change in $\mathrm{VAF}_{\text {total }}$ when the cross-validation was applied within conventional walking (see methods) and we found a threshold of $4.9 \%$. This value was similar to the value $(6.1 \%)$ found by a previous study using a similar approach [55]. Considering this threshold, muscle synergies were affected by Nordic walking (decrease of $\mathrm{VAF}_{\text {total }}>4.9 \%$ ) in all participants.

The cross-reconstruction of Nordic walking EMG pattern using the synergy vectors obtained from conventional walking was more problematic. Indeed, this cross-reconstruction showed bad quality $\left(\mathrm{VAF}_{\text {total }} 74.4 \pm 8.2 \%\right)$. This was an expected result, since in conventional walking the upper limb muscles were activated very low [40] and thus the representativeness of their weightings in the overall synergies composition was low [23, 27, 32]. In spite of these observations, we decided not to consider the results of this cross-reconstruction as a measure of robustness of the muscle synergies across conditions. As occurred in the original signals, the $\mathrm{VAF}_{\text {muscle }}$ of lower limb muscles showed overall high $\operatorname{VAF}_{\text {muscle }}$ values $(\geq 86 \%)$, while trunk ad upper limb muscles showed lower values (ranging from $68 \%$ to $80 \%$, see Figure 5). These results further underlined that reconstructing the EMG envelopes of trunk and upper limb muscles could be problematic. However, the $\mathrm{VAF}_{\text {muscle }}$ indexes were greater than $75 \%$ for all muscles except ES, LD, and BB (Figure 5), suggesting that most muscles were well represented in the cross-reconstruction.

This article is protected by copyright. All rights reserved. 


\section{Analysis on a subset of muscles}

As mentioned before, synergy extraction becomes challenging when muscles with low EMG signal-to-noise ratio are present. This was the case of upper limb muscles during conventional walking). To check whether the low-quality cross-reconstruction from Nordic walking to conventional walking was only due to this matter, we re-analysed only a subset data of muscles. Thus, we extracted the synergies of 10 out of 15 muscles, removing the five upper limb muscles ( $\mathrm{LD}, \mathrm{AD}, \mathrm{PD}, \mathrm{BB}, \mathrm{TB})$ from the analysis. Considering the criteria mentioned above, five synergies were necessary in this analysis for both conventional walking and Nordic walking. In this case, no difference in $\mathrm{VAF}_{\text {total }}$ between the two tasks was observed (conventional walking $94.0 \pm 1.0 \%$, Nordic walking $93.5 \pm 1.0 \%, \mathrm{p}=0.335$, see Table 2).

When the synergy vectors obtained from Nordic walking was used to reconstruct the conventional walking, the $\mathrm{VAF}_{\text {total }}$ was very high $(88.9 \pm 2.9 \%)$. Thus, the cross-validation provided accurate reconstruction results $\mathrm{VAF}_{\text {total }}>80 \%$ in all subjects (see Table 2). The threshold for considering a change in VAF as a change in muscle synergies was $4.7 \%$ (that is the upper limit of the $95 \%$ of Confidence Interval of the within-task variability, see methods). Considering this threshold, muscle synergies were affected by Nordic walking (decrease of $\mathrm{VAF}_{\text {total }}>4.0 \%$ ) in only two out of nine participants.

\section{DISCUSSION}

We compared the muscle synergies identified in Nordic walking and conventional walking in a group of experienced Nordic walkers. Nordic walking did not require more muscle synergies than conventional walking in most of the participants. This finding suggests that the motor control of Nordic walking was not more complex than that required for conventional walking. The two types of locomotion shared four out five muscle synergies, specifically those related to the activation of lower limb muscles. Synergy \#5 mainly involved the activation of upper limb muscles and was task-specific, having its main role during upper limb propulsive action in Nordic walking, while during arm swing when conventional walking.

\section{Muscle activation}

The herein findings confirm the results of previous investigations that showed an overall higher activation of upper body muscles in Nordic walking than in conventional walking $[9,10]$. In particular, upper trapezius, latissimus dorsi, posterior deltoid, biceps

This article is protected by copyright. All rights reserved. 
brachii, and triceps brachii were on average more activated during Nordic walking, when the poles are used (see Figure 1). Interestingly, latissimus dorsi, posterior deltoid, and triceps brachii, which are the muscles deputed to the propulsive phase trough the poling activation, showed a more pronounced peak of activation in Nordic walking compared to conventional walking (Figure 1). Table 1 shows that these muscles were activated later (by 12-17\%) in the cycle phase in Nordic walking with respect to conventional walking. This particular activation pattern of walking with the pole was likely due to the Nordic walking technique, that requires a long poling phase. The higher activation of these muscles is likely the main cause of the augmented energy expenditure of Nordic walking with respect to conventional walking [10]. An increase in activation of anterior deltoid and biceps brachii muscles during Nordic walking was also seen, not only during the pole recovery but also the poling phase, when they are co-activated with the triceps brachii and latissimus dorsii muscles, to stabilise the elbow and shoulder joints [10].

In line with a previous study [10], lower-limb muscles showed a similar involvement during conventional walking and Nordic walking, both regarding the level and the shape of activation (Figure 1 and Table 1). The only exception was the rectus femoris muscles. This muscle has been reported to have two peaks of activation during walking: the first peak usually coincides with the stance phase and the second peak coincides with the swing phase [65]. In Nordic walking the second peak seemed to be lowered with respect to walking (Figure 1), suggesting that the use of poles decreased the intervention of this hip-flexor muscle in the swing phase (60-70\% of gait cycle). This change seems also to induce a change in activation profile of synergy \#2 (see "Comparison between muscle synergies").

\section{Dimensionality}

Our results show that five muscle synergies accounted for $\approx 91 \%$ of EMG pattern variance in a large number of muscles that are active during Nordic walking, including those of the legs, trunk, and upper body [25, 32]. Thus, in experienced Nordic walkers, central nervous system could accomplish the control of this particular form of four-limbs locomotion by means of a small set of muscle synergies.

Contrary to the expectations, Nordic walking did not require more synergies than conventional walking. When we compared the individual results, three subjects out of nine showed one more synergy, and one subject showed one less synergy in Nordic walking compared to conventional walking. Thus, we can state that in most subjects the inclusion of upper limbs with a propulsive function did not increase the complexity of motor coordination

This article is protected by copyright. All rights reserved. 
of walking. Since the upper body is cyclically active in Nordic walking with the same frequency of leg it becomes part of the locomotion, without increasing the complexity of motor control of walking.

As there is an increasing use of Nordic walking as physical exercise for health, this finding is valuable when considering the clinical perspective. Nordic walking has been adopted in a wide range of disease, including those affecting neurological functions (e.g. Parkinson) [7, 8]. The fact that in our sample Nordic walking was not more complex than conventional walking, suggests that Nordic walking is suitable in adapted physical activity programs. However, these results may not be applied to novice subjects with lower skill level than our participants. Indeed, our participants were Nordic walking instructors with an apparently consolidated Nordic walking technique and at least two years of practice. This is an important point because the correct technique of Nordic walking may necessitate of a sufficient period of practice to be learned. Thus, future studies should address the question whether the coordinative organization of Nordic walking would be the same also for unexperienced individuals or even neurologically impaired patients.

Moreover, it should be taken into account that the Nordic walking technique might not be consistent across participants. Indeed, despite all participants tried to follow the Nordic walking technique guidelines, each participant might have different personal technical interpretation of the Nordic walking technique. It is possible that less skilled subjects would show even more differentiated muscle coordination.

On average, the activation of all muscles was well represented by the five muscle synergies in both tasks (Figure 3). The five synergies showed sequential activation in the gait cycle, with each synergy referring to a specific subtask of the gait cycle, in both locomotion modes. However, upper limb muscles showed low $\mathrm{VAF}_{\text {muscle }}$ values in conventional walking. This is likely due to the low EMG signal-to-noise ratio of upper limb muscles that reduced the representativeness of these muscles in the conventional walking [23, 27, 32, 40]. Conversely, upper limb muscles showed high $\mathrm{VAF}_{\text {muscle }}$ values in Nordic walking, likely because of higher EMG signal-to-noise ratio.

\section{Comparison between muscle synergies}

Considering the similarity indexes showed in Table 3, it is possible to say that the four muscle synergies mainly activating the lower limbs are similar in the two forms of locomotion. Indeed, the normalized scalar product between the synergy vectors were high $(\geq 0.81)$ for the synergies $\# 1, \# 2$, \#3 and \#4. These results suggest that Nordic walking share 
the same muscle synergies of conventional walking if trunk and lower limb muscles are considered. Thus walking with the use of poles does not change the spatial organization of conventional walking.

Considering the profile and the magnitude of activation of each synergy, it is possible to identify the role of each synergy within the locomotion cycle. All the five extracted muscle synergies can be related to specific subtasks of gait cycle, in both conditions (Figure 4). The muscle synergies \#1, \#2, \#3, and \#4 were activated with the same profiles in the two tasks and thus were considered robust across conditions. Their role in both types of locomotion was "leg deceleration", "weight acceptance" and "single leg support", "leg propulsion", "trunk extension" and "arm swing" respectively (see results for details). This means that, at least regarding these four synergies, participants did not change the temporal organization in the mechanics of walking with and without the use of poles.

Despite the between-tasks similarity of muscle synergies from \#1, \#2, \#3 and \#4, the activations of hip muscles presented some changes within synergies. During Nordic walking the gluteus medius seems to be less active in the single leg support synergy (\#2) compared to conventional walking. This suggests that walking with poles might help in stabilizing the hip consequently inducing a possible less activation of this muscle. Regarding RF, it is possible to see that its lower activation during the swing phase (see Fig 1, second peak of RF activation about $60-70 \%$ of gait cycle) resulted in lower activation coefficients of synergy \#2 during the same phase in Nordic walking compared to conventional walking (Fig 4). Moreover, biceps femoris and semitendinosus muscles seemed to be less activated in the leg deceleration synergy (\#1). Together, these results may suggest that the leg swing and single leg support functions recruited the hip muscles less in Nordic walking compared to conventional walking.

The functional role, muscle composition and shape of activation of synergy \#5 was different in the two locomotion modes (Figure 4 and Table 3). This synergy mainly involved the activation of upper limb muscles, that in conventional walking are involved in the "upper limb swing". However, these muscles have very low activation in conventional walking. In Nordic walking this synergy was clearly involved in the poling phase that is the "upper limb propulsion". The main important differences were that the involvement of shoulder and elbow extensors, as latissimus dorsi, posterior deltoid and triceps brachii, in this synergy was relatively higher in Nordic walking than in conventional walking. Beyond the peculiar activation of shoulder and elbow extensors, the activation shoulder and elbow flexors (anterior deltoid and biceps brachii together with the upper trapezius acting as stabilizer)

This article is protected by copyright. All rights reserved. 
was spread differently across synergies. While these muscles are more activated in synergy \#5 of conventional walking, they are more activated in the synergy \#1 of Nordic walking. Thus, Nordic walking leads to a change in the muscles coordination for the upper limbs. Taking together, these results suggest that the use of the poles modified the spatial coordination and the magnitude of activation of the synergy involved in the poling action, thus requiring a task-specific muscle synergy.

\section{Cross-validation of the extracted muscle synergies}

The comparison of muscle synergies between different tasks might not exclusively be based on similarity indexes but should also rely on cross-validation method [38]. The use of a cross-validation method, such as fixing the muscle weightings in combination with the activation coefficients to be reconstructed, was found to be more valuable than simply comparing muscle weightings [38]. In this study, we reconstructed the conventional walking EMGs using the muscle synergy vectors obtained in Nordic walking. It can be assumed that if the EMG pattern of one task can be reconstructed by using the vectors of another task, then the two tasks share the same modules [34]. In the present study, we found that the crossreconstruction of conventional walking EMG pattern accounted for $79.6 \%$ of total VAF (Table 2). A value of $80 \%$ is commonly accepted as a threshold to define a good reconstruction. Looking at individual responses, five subjects out of nine showed VAF of cross-reconstruction higher that $80 \%$ (Table 2). Consequently, we can state that it was possible to use the muscle synergies of Nordic walking to describe the muscular activation pattern of conventional walking in roughly half of subjects.

Beyond the overall good cross-reconstruction quality, we furthermore investigate if the change in VAF between tasks was higher than the change in VAF calculated within the same task. Thus, we firstly measured the robustness of cross-reconstruction within conventional walking (see methods). Then we evaluated the between-task individual changes in total VAF with respect to the within-task variability [55, 63]. Thus, we considered that the synergies of conventional walking were significantly affected by Nordic walking if the total VAF was reduced more than within-task variability. Considering this threshold (4.9\%), we found that in all subjects the change in VAF between tasks was higher than within task. Thus, using this criteria in all subjects the muscle synergies of Nordic walking were somewhat different than those used in conventional walking. Again, the two criteria used to determine the quality of the cross-reconstruction provided different findings for five subjects out of 
nine. It is reasonable to consider the second criterion, i.e. change in VAF greater than withintask variability, more sensitive that the first criterion, i.e. overall $\mathrm{VAF}>80 \%$.

Since the synergy that showed most differences between tasks was related to the activation of upper limb muscles, we furthermore wanted to investigate if removing the upper limbs from the analysis would affect the results of cross-reconstruction. Thus, we performed the cross-reconstruction again using only lower limb and trunk muscles, removing from the analysis the upper limbs. In such a way, the goodness of cross-reconstruction was high for all subjects (all VAF>84\%) and the between-tasks change in VAF was lower than within-task variability in seven out of nine subjects (Table 2). This means that the low VAF of crossreconstruction when using the whole set of muscles was mostly caused by differences in upper limb and less by differences in lower limb and trunk. Together these findings suggest that regarding trunk lower and body muscles, Nordic walking shares the same muscle synergies with conventional walking.

\section{Conclusions}

We can conclude that, contrary to our hypothesis, in experienced individuals Nordic walking does not necessitate of more complex movement organization than conventional walking. Moreover, Nordic walking and conventional walking share the muscle synergies mainly involving the activation of lower limb and trunk muscles. Therefore, walking with the use of poles does not profoundly change the muscle coordination of lower body. However, the poling action modify the spatial and temporal structure of the muscle synergy involved in this action, that represents the upper body action. Thus, Nordic walking requires a taskspecific muscle synergy for the sub-task of upper limb propulsion, but not for trunk and lower limb action.

\section{FUTURE PERSPECTIVES}

Since the Nordic walking did not alter the muscle coordination of lower limbs, we can suggest the it can be suitable to be adopted in adapted physical activity programs. However, a sufficient period of practice is necessary to learn the technique. Future studies should characterize the learning period of Nordic walking and investigate whether muscle synergies may reflect different level of expertise in Nordic walking. 


\section{ACKNOWLEDGMENTS}

Authors would thank Raffaela Rosa from ANWI (Associazione Nordic Walking

Italia) for her help and the participants who took part in the study.

\section{REFERENCES}

1. Fritschi, J.O., W.J. Brown, R. Laukkanen, and J.G.Z. van Uffelen, The effects of pole walking on health in adults: A systematic review. Scandinavian Journal of Medicine \& Science in Sports, 2012. 22(5): p. e70-e78.

2. Mathieson, S. and C.W.C. Lin, Health benefits of Nordic walking; a systematic review. British Journal of Sports Medicine, 2014. 48(21): p. 1577-1578.

3. Parkatti, T., J. Perttunen, and P. Wacker, Improvements in Functional Capacity From Nordic Walking: A Randomized Controlled Trial Among Older Adults. Journal of Aging and Physical Activity, 2012. 20(1): p. 93-105.

4. Schena, F.F., H. Figard-Fabre, N. Fabre, and A. Leonardi, Specifc Effects of Nordic Walking Training In Obese Middle-aged Women. Medicine and Science in Sports and Exercise, 2010. 42(5): p. 597-598.

5. Figard-Fabre, H., N. Fabre, A. Leonardi, and F. Schena, Efficacy of Nordic walking in obesity management. Int J Sports Med, 2011. 32(6): p. 407-14.

6. Figard-Fabre, H., N. Fabre, A. Leonardi, and F. Schena, Physiological and perceptual responses to Nordic walking in obese middle-aged women in comparison with the normal walk. Eur J Appl Physiol, 2010. 108(6): p. 1141-51.

7. Monteiro, E.P., L.T. Franzoni, D.M. Cubillos, A. de Oliveira Fagundes, A.R. Carvalho, H.B. Oliveira, P.D. Pantoja, F.B. Schuch, C.R. Rieder, F.G. Martinez, and L.A. Peyré-Tartaruga, Effects of Nordic walking training on functional parameters in Parkinson's disease: a randomized controlled clinical trial. Scandinavian Journal of Medicine \& Science in Sports, 2016: p. n/a-n/a.

8. Bombieri, F., F. Schena, B. Pellegrini, P. Barone, M. Tinazzi, and R. Erro, Walking on four limbs: A systematic review of Nordic Walking in Parkinson disease. Parkinsonism Relat Disord, 2017. 38: p. 8-12.

9. Schiffer, T., A. Knicker, M. Montanarella, and H.K. Struder, Mechanical and physiological effects of varying pole weights during Nordic walking compared to walking. Eur J Appl Physiol, 2011. 111(6): p. 1121-6.

10. Pellegrini, B., L.A. Peyre-Tartaruga, C. Zoppirolli, L. Bortolan, E. Bacchi, H. Figard-Fabre, and F. Schena, Exploring Muscle Activation during Nordic Walking: A Comparison between Conventional and Uphill Walking. PLoS One, 2015. 10(9): p. e0138906.

11. Shim, J.M., H.Y. Kwon, H.R. Kim, B.I. Kim, and J.H. Jung, Comparison of the Effects of Walking with and without Nordic Pole on Upper Extremity and Lower Extremity Muscle Activation. J Phys Ther Sci, 2013. 25(12): p. 1553-6.

12. Schiffer, T., A. Knicker, U. Hoffman, B. Harwig, W. Hollmann, and H.K. Struder, Physiological responses to nordic walking, walking and jogging. Eur J Appl Physiol, 2006. 98(1): p. 56-61.

13. Hansen, E.A. and G. Smith, Energy expenditure and comfort during Nordic walking with different pole lengths. J Strength Cond Res, 2009. 23(4): p. 1187-94.

14. Sugiyama, K., M. Kawamura, H. Tomita, and S. Katamoto, Oxygen uptake, heart rate, perceived exertion, and integrated electromyogram of the lower and upper extremities during level and Nordic walking on a treadmill. J Physiol Anthropol, 2013. 32(1): p. 2.

15. Bieler, T., V. Siersma, S.P. Magnusson, M. Kjaer, H.E. Christensen, and N. Beyer, In hip osteoarthritis, Nordic Walking is superior to strength training and home-based exercise for improving function. Scandinavian Journal of Medicine \& Science in Sports, 2016: p. n/a-n/a.

This article is protected by copyright. All rights reserved. 
16. Kortas, J., K. Prusik, D. Flis, K. Prusik, E. Ziemann, N. Leaver, and J. Antosiewicz, Effect of Nordic Walking training on iron metabolism in elderly women. Clinical Interventions in Aging, 2015. 10: p. 1889-1896.

17. Hug, F., Can muscle coordination be precisely studied by surface electromyography? J Electromyogr Kinesiol, 2011. 21(1): p. 1-12.

18. Raasch, C.C. and F.E. Zajac, Locomotor strategy for pedaling: muscle groups and biomechanical functions. J Neurophysiol, 1999. 82(2): p. 515-25.

19. Tresch, M.C., P. Saltiel, and E. Bizzi, The construction of movement by the spinal cord. Nat Neurosci, 1999. 2(2): p. 162-7.

20. d'Avella, A., P. Saltiel, and E. Bizzi, Combinations of muscle synergies in the construction of a natural motor behavior. Nat Neurosci, 2003. 6(3): p. 300-8.

21. Cheung, V.C., A. d'Avella, M.C. Tresch, and E. Bizzi, Central and sensory contributions to the activation and organization of muscle synergies during natural motor behaviors. J Neurosci, 2005. 25(27): p. 6419-34.

22. Bizzi, E., V.C. Cheung, A. d'Avella, P. Saltiel, and M. Tresch, Combining modules for movement. Brain Res Rev, 2008. 57(1): p. 125-33.

23. Tresch, M.C., V.C. Cheung, and A. d'Avella, Matrix factorization algorithms for the identification of muscle synergies: evaluation on simulated and experimental data sets. J Neurophysiol, 2006. 95(4): p. 2199-212.

24. Tresch, M.C. and A. Jarc, The case for and against muscle synergies. Curr Opin Neurobiol, 2009. 19(6): p. 601-7.

25. Ivanenko, Y.P., R.E. Poppele, and F. Lacquaniti, Five basic muscle activation patterns account for muscle activity during human locomotion. J Physiol, 2004. 556(Pt 1): p. 267-82.

26. Gui, K. and D. Zhang, Influence of locomotion speed on biomechanical subtask and muscle synergy. J Electromyogr Kinesiol, 2016. 30: p. 209-15.

27. Gizzi, L., J.F. Nielsen, F. Felici, Y.P. Ivanenko, and D. Farina, Impulses of activation but not motor modules are preserved in the locomotion of subacute stroke patients. J Neurophysiol, 2011. 106(1): p. 202-10.

28. Ivanenko, Y.P., R.E. Poppele, and F. Lacquaniti, Motor control programs and walking. Neuroscientist, 2006. 12(4): p. 339-48.

29. Monaco, V., A. Ghionzoli, P. Dario, and S. Micera, Muscle synergies during walking: comparison between young and elderly people. Preliminary results. Conf Proc IEEE Eng Med Biol Soc, 2008. 2008: p. 5370-3.

30. Danna-Dos-Santos, A., A.M. Degani, and M.L. Latash, Flexible muscle modes and synergies in challenging whole-body tasks. Exp Brain Res, 2008. 189(2): p. 171-87.

31. de Rugy, A., G.E. Loeb, and T.J. Carroll, Are muscle synergies useful for neural control? Frontiers in Computational Neuroscience, 2013. 7.

32. Cappellini, G., Y.P. Ivanenko, R.E. Poppele, and F. Lacquaniti, Motor patterns in human walking and running. J Neurophysiol, 2006. 95(6): p. 3426-37.

33. Gonzalez-Vargas, J., M. Sartori, S. Dosen, D. Torricelli, J.L. Pons, and D. Farina, A predictive model of muscle excitations based on muscle modularity for a large repertoire of human locomotion conditions. Front Comput Neurosci, 2015. 9: p. 114.

34. Yokoyama, H., T. Ogawa, N. Kawashima, M. Shinya, and K. Nakazawa, Distinct sets of locomotor modules control the speed and modes of human locomotion. Scientific Reports, 2016. 6.

35. Zeni, J.A., Jr., J.G. Richards, and J.S. Higginson, Two simple methods for determining gait events during treadmill and overground walking using kinematic data. Gait Posture, 2008. 27(4): p. 710-4.

36. Hermens, H.J., B. Freriks, C. Disselhorst-Klug, and G. Rau, Development of recommendations for SEMG sensors and sensor placement procedures. J Electromyogr Kinesiol, 2000. 10(5): p. 361-74.

This article is protected by copyright. All rights reserved. 
37. Beretta Piccoli, M., A. Rainoldi, C. Heitz, M. Wuthrich, G. Boccia, E. Tomasoni, C. Spirolazzi, M. Egloff, and M. Barbero, Innervation zone locations in 43 superficial muscles: toward a standardization of electrode positioning. Muscle Nerve, 2014. 49(3): p. 413-21.

38. Oliveira, A.S., L. Gizzi, D. Farina, and U.G. Kersting, Motor modules of human locomotion: influence of EMG averaging, concatenation, and number of step cycles. Front Hum Neurosci, 2014. 8: p. 335.

39. Willigenburg, N.W., A. Daffertshofer, I. Kingma, and J.H. van Dieen, Removing ECG contamination from EMG recordings: a comparison of ICA-based and other filtering procedures. J Electromyogr Kinesiol, 2012. 22(3): p. 485-93.

40. Shiavi, R., C. Frigo, and A. Pedotti, Electromyographic signals during gait: criteria for envelope filtering and number of strides. Med Biol Eng Comput, 1998. 36(2): p. 171-8.

41. Hug, F., N.A. Turpin, A. Couturier, and S. Dorel, Consistency of muscle synergies during pedaling across different mechanical constraints. J Neurophysiol, 2011. 106(1): p. 91-103.

42. Barroso, F.O., D. Torricelli, J.C. Moreno, J. Taylor, J. Gomez-Soriano, E. Bravo-Esteban, S. Piazza, C. Santos, and J.L. Pons, Shared muscle synergies in human walking and cycling. J Neurophysiol, 2014. 112(8): p. 1984-98.

43. Ivanenko, Y.P., G. Cappellini, N. Dominici, R.E. Poppele, and F. Lacquaniti, Coordination of locomotion with voluntary movements in humans. J Neurosci, 2005. 25(31): p. 7238-53.

44. Torres-Oviedo, G., J.M. Macpherson, and L.H. Ting, Muscle synergy organization is robust across a variety of postural perturbations. J Neurophysiol, 2006. 96(3): p. 1530-46.

45. Clark, D.J., L.H. Ting, F.E. Zajac, R.R. Neptune, and S.A. Kautz, Merging of healthy motor modules predicts reduced locomotor performance and muscle coordination complexity poststroke. J Neurophysiol, 2010. 103(2): p. 844-57.

46. Lee, D. and H. Seung, Algorithms for non-negative matrix factorization. Adv Neurol, 2001(13): p. 556-562.

47. Lee, D.D. and H.S. Seung, Learning the parts of objects by non-negative matrix factorization. Nature, 1999. 401(6755): p. 788-91.

48. Dominici, N., Y.P. Ivanenko, G. Cappellini, A. d'Avella, V. Mondi, M. Cicchese, A. Fabiano, T. Silei, A. Di Paolo, C. Giannini, R.E. Poppele, and F. Lacquaniti, Locomotor primitives in newborn babies and their development. Science, 2011. 334(6058): p. 997-9.

49. Torres-Oviedo, G. and L.H. Ting, Muscle synergies characterizing human postural responses. J Neurophysiol, 2007. 98(4): p. 2144-56.

50. Ambrosini, E., C. De Marchis, A. Pedrocchi, G. Ferrigno, M. Monticone, M. Schmid, T. D'Alessio, S. Conforto, and S. Ferrante, Neuro-Mechanics of Recumbent Leg Cycling in PostAcute Stroke Patients. Ann Biomed Eng, 2016. 44(11): p. 3238-3251.

51. De Marchis, C., M. Schmid, D. Bibbo, I. Bernabucci, and S. Conforto, Inter-individual variability of forces and modular muscle coordination in cycling: a study on untrained subjects. Hum Mov Sci, 2013. 32(6): p. 1480-94.

52. Nishida, K., S. Hagio, B. Kibushi, T. Moritani, and M. Kouzaki, Comparison of muscle synergies for running between different foot strike patterns. PLoS One, 2017. 12(2): p. e0171535.

53. Janshen, L., A. Santuz, A. Ekizos, and A. Arampatzis, Modular control during incline and level walking in humans. J Exp Biol, 2017. 220(Pt 5): p. 807-813.

54. Hug, F., N.A. Turpin, A. Guevel, and S. Dorel, Is interindividual variability of EMG patterns in trained cyclists related to different muscle synergies? J Appl Physiol, 2010. 108(6): p. 172736.

55. van den Hoorn, W., P.W. Hodges, J.H. van Dieen, and F. Hug, Effect of acute noxious stimulation to the leg or back on muscle synergies during walking. J Neurophysiol, 2015. 113(1): p. 244-54.

56. Martino, G., Y.P. Ivanenko, A. d'Avella, M. Serrao, A. Ranavolo, F. Draicchio, G. Cappellini, C. Casali, and F. Lacquaniti, Neuromuscular adjustments of gait associated with unstable conditions. J Neurophysiol, 2015. 114(5): p. 2867-82.

This article is protected by copyright. All rights reserved. 
57. Turpin, N.A., A. Guevel, S. Durand, and F. Hug, Fatigue-related adaptations in muscle coordination during a cyclic exercise in humans. J Exp Biol, 2011. 214(Pt 19): p. 3305-14.

58. Torres-Oviedo, G. and L.H. Ting, Subject-specific muscle synergies in human balance control are consistent across different biomechanical contexts. J Neurophysiol, 2010. 103(6): p. 3084-98.

59. Cheung, V.C., L. Piron, M. Agostini, S. Silvoni, A. Turolla, and E. Bizzi, Stability of muscle synergies for voluntary actions after cortical stroke in humans. Proc Natl Acad Sci U S A, 2009. 106(46): p. 19563-8.

60. Oliveira, A.S., L. Gizzi, S. Ketabi, D. Farina, and U.G. Kersting, Modular Control of Treadmill vs Overground Running. PLoS One, 2016. 11(4): p. e0153307.

61. Gizzi, L., S. Muceli, F. Petzke, and D. Falla, Experimental Muscle Pain Impairs the Synergistic Modular Control of Neck Muscles. PLoS One, 2015. 10(9): p. e0137844.

62. Heales, L.J., F. Hug, D.A. MacDonald, B. Vicenzino, and P.W. Hodges, Is synergistic organisation of muscle coordination altered in people with lateral epicondylalgia? A casecontrol study. Clin Biomech (Bristol, Avon), 2016. 35: p. 124-31.

63. Frere, J. and F. Hug, Between-subject variability of muscle synergies during a complex motor skill. Front Comput Neurosci, 2012. 6: p. 99.

64. Steele, K.M., M.C. Tresch, and E.J. Perreault, The number and choice of muscles impact the results of muscle synergy analyses. Front Comput Neurosci, 2013. 7: p. 105.

65. Winter, D.A. and H.J. Yack, EMG profiles during normal human walking: stride-to-stride and inter-subject variability. Electroencephalogr Clin Neurophysiol, 1987. 67(5): p. 402-11.

\section{CAPTIONS}

Figure 1 - Group-averaged envelopes (with 95\% of Confidence Interval) of electromyographic signals are reported for 15 recorded muscles while walking (blue) and Nordic walking (red). Each represented pattern consists in the average of 15 to 25 cycles of the nine individuals (total about 180 cycles).

Figure 2 - Variability accounted for $\left(\mathrm{VAF}_{\text {total }}\right)$ individual values (grey lines) and mean (black line) based on the number of the extracted synergies with the nonnegative matrix factorization (NNMF) for walking (left panel) and Nordic walking (right panel).

Figure 3 - Individual (circles) and average (bar) variability accounted for values $($ mean $\pm S D)$ for each muscle $\left(\mathrm{VAF}_{\text {muscle }}\right)$ for walking (left panel) and Nordic walking (right panel). A minimum value of $75 \%$ for $\mathrm{VAF}_{\text {muscle }}$ was used to consider the quality of reconstruction of each muscle good.

Figure 4 - Temporal activation coefficients (with 95\% CI, left panel) and muscle synergy vectors (right panel) are reported for conventional walking (blue) and Nordic walking (red). The five synergies extracted are designated in chronological order (with respect to the timing of the main peak of activation coefficients) and numbered from \#1 to \#5.

This article is protected by copyright. All rights reserved. 
Figure 5 - Individual (circles) and average (bar) variability accounted for $\mathrm{VAF}_{\text {muscle }}$ values are reported when nonnegative matrix factorization (NNMF) was applied to crossreconstruct the EMG patterns of conventional walking $(\mathrm{CW})$ using the synergy vectors obtained in Nordic walking (NW).

Table 1 - Cross-correlation between EMG envelopes of muscles

$\begin{array}{lrlrl} & \text { cross-correlation }\left(r_{\text {max }}\right) & \text { Lag }(\%) & \text { WA vs NW } \\ & \text { Mean } & \text { SD } & \text { mean } & \text { SD } \\ \text { TA } & 0.95 & 0.02 & 0.00 & 1 \\ \text { SO } & 0.98 & 0.01 & 0.00 & 1 \\ \text { GM } & 0.97 & 0.02 & 0.00 & 2 \\ \text { VL } & 0.98 & 0.02 & 0.00 & 1 \\ \text { RF } & 0.91 & 0.09 & -1.00 & 1 \\ \text { BF } & 0.94 & 0.04 & 0.00 & 2 \\ \text { ST } & 0.92 & 0.07 & 0.00 & 1 \\ \text { Glu } & 0.96 & 0.03 & 0.00 & 1 \\ \text { UT } & 0.86 & 0.05 & 0.00 & 3 \\ \text { ES } & 0.88 & 0.16 & -1.00 & 2 \\ \text { LD } & 0.85 & 0.09 & -17.00 & 15^{*} \\ \text { AD } & 0.86 & 0.08 & 1.00 & 2 \\ \text { PD } & 0.92 & 0.02 & -12.00 & 6^{*} \\ \text { BB } & 0.85 & 0.04 & -4.00 & 5 \\ \text { TB } & 0.89 & 0.06 & -12.00 & 8^{*}\end{array}$

The cross-correlation coefficients $r_{\max }($ mean $\pm \mathrm{SD})$ were calculated within each muscle, for each subject, between the two forms of locomotion. $r_{\max }$ provides an indication of the similarity between the waveforms of envelopes. Negative values of lags shows that the peak of conventional walking $(\mathrm{CW})$ envelopes occurs before the peak of Nordic walking (NW) envelopes.

This article is protected by copyright. All rights reserved. 
Table 2 - Results on the number of muscle synergy dimensionality and cross-reconstruction validation

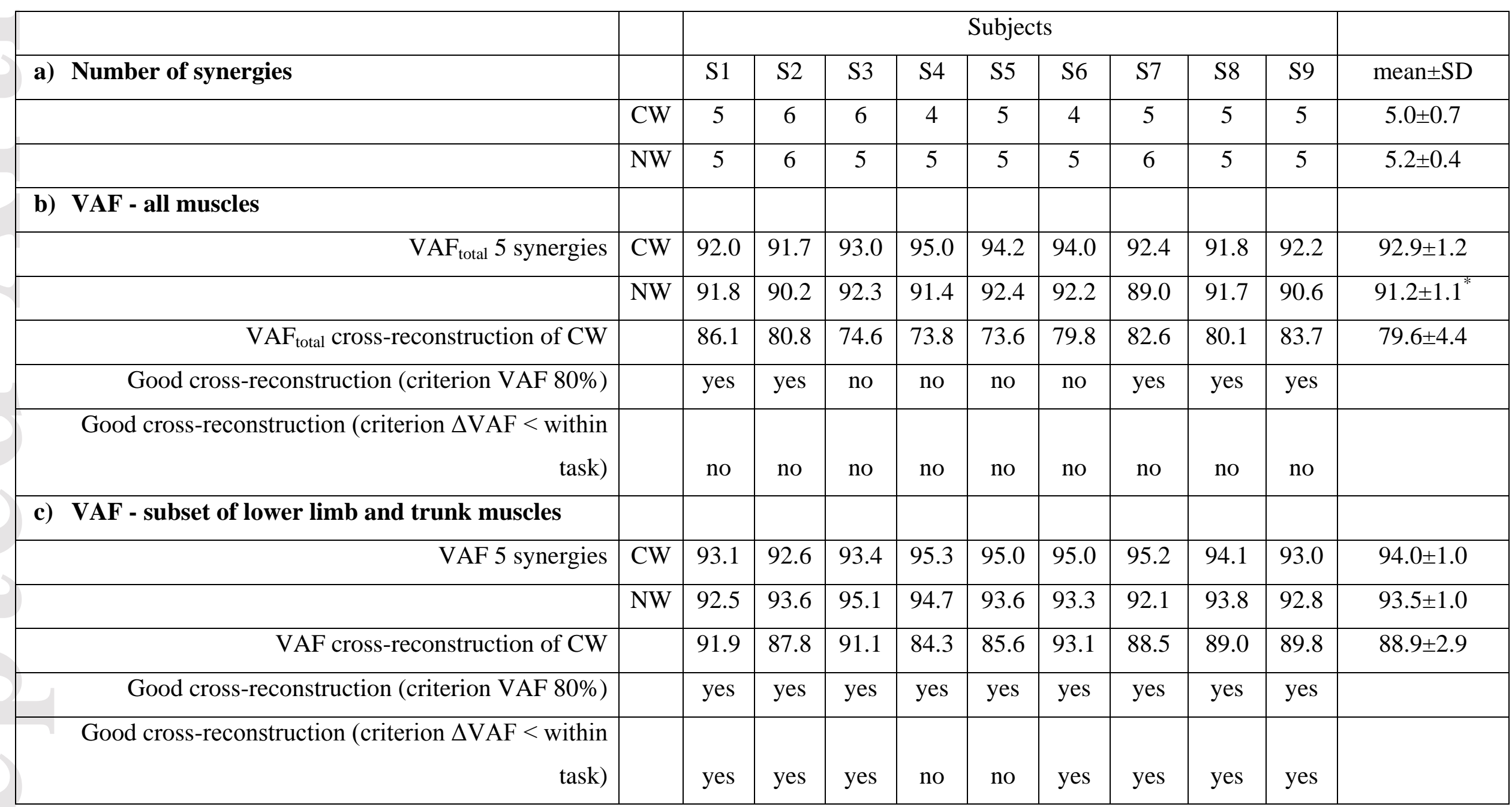

This article is protected by copyright. All rights reserved. 
For each subject (from S1 to S9), in the part a) the number of synergies extracted are reported. The parts b) provide VAF results for the analysis conducted on all 15 muscles while part c) for the subset of 10 lower limb and trunk muscles. In particular, the VAF explained by five synergies are reported for CW and NW. The VAF obtained when the EMG of CW are cross-reconstructed using the synergy vector of NW is also reported. Then, for each subject, is reported whether (yes/no) the cross-reconstruction provided good results based on two criteria: i) the VAF of crossreconstruction is greater than $80 \%$ (VAF $80 \%)$ and ii) if the between-task change in VAF is less the within-task variability $(\triangle \mathrm{VAF}<$ within task), see method for further details. Statistically significant differences between CW and NW are reported as * $\mathrm{p}<0.05$. Variance accounted for (VAF); conventional walking (CW); Nordic walking (NW); Electromyography (EMG).

This article is protected by copyright. All rights reserved. 
Table 3 - Similarities of muscle synergies between conventional walking and Nordic walking

\begin{tabular}{|c|c|c|c|c|c|c|c|}
\hline & \multicolumn{6}{|c|}{ Activation coefficients } & \multirow{3}{*}{$\begin{array}{c}\text { Synergy vectors } \\
\text { Normalized scalar } \\
\text { product }\end{array}$} \\
\hline & \multirow{2}{*}{$\begin{array}{c}\text { Cross-correlation } \\
\qquad\left(r_{\max }\right)\end{array}$} & \multirow{2}{*}{$\begin{array}{c}\text { Lag } \\
(\% \text { cycle })\end{array}$} & \multicolumn{2}{|c|}{ FWHM (a.u.) } & \multicolumn{2}{|c|}{$\begin{array}{l}\text { Duration of FWHM } \\
\qquad(\% \text { cycle })\end{array}$} & \\
\hline & & & $\mathrm{CW}$ & NW & $\mathrm{CW}$ & NW & \\
\hline Synergy \#1 & $0.91 \pm 0.13$ & $0 \pm 2$ & $1.29 \pm 0.37$ & $1.25 \pm 0.16$ & $16 \pm 5$ & $15 \pm 3$ & $0.85 \pm 0.08$ \\
\hline Synergy \#2 & $0.96 \pm 0.09$ & $0 \pm 1$ & $1.39 \pm 0.54$ & $1.39 \pm 0.11$ & $12 \pm 2$ & $12 \pm 2$ & $0.88 \pm 0.08$ \\
\hline Synergy \#3 & $0.98 \pm 0.03$ & $0 \pm 1$ & $1.26 \pm 0.42$ & $1.03 \pm 0.15$ & $19 \pm 7$ & $21 \pm 8$ & $0.95 \pm 0.03$ \\
\hline Synergy \#4 & $0.93 \pm 0.03$ & $0 \pm 1$ & $0.99 \pm 0.45$ & $0.91 \pm 0.22$ & $14 \pm 9$ & $15 \pm 6$ & $0.81 \pm 0.10$ \\
\hline Synergy \#5 & $0.81 \pm 0.13$ & $2 \pm 3$ & - & - & - & - & $0.55 \pm 0.23$ \\
\hline
\end{tabular}

For each extracted synergy the following parameters are reported (mean $\pm \mathrm{SD}$ ): the cross-correlation coefficients $\mathrm{r}_{\text {max }}$ which provide an index of similarity between the waveforms of synergy coefficients $\mathbf{C}$; lags (time shift) between the peak of the activation coefficient (negative values meaning that the peak in Nordic walking (NW) occurs later than in conventional walking (CW); duration of the burst activation of the synergy coefficient defined as the full-width at the half maximum (FWHM) of the main peak; magnitude of the activation calculated as the root mean square of the activity during the FWHM; Normalized scalar product between synergy vectors W (muscle weightings). The FWHM and duration of FWHM were not calculated for synergy \#5 because the were different in the two conditions (see methods).

This article is protected by copyright. All rights reserved. 

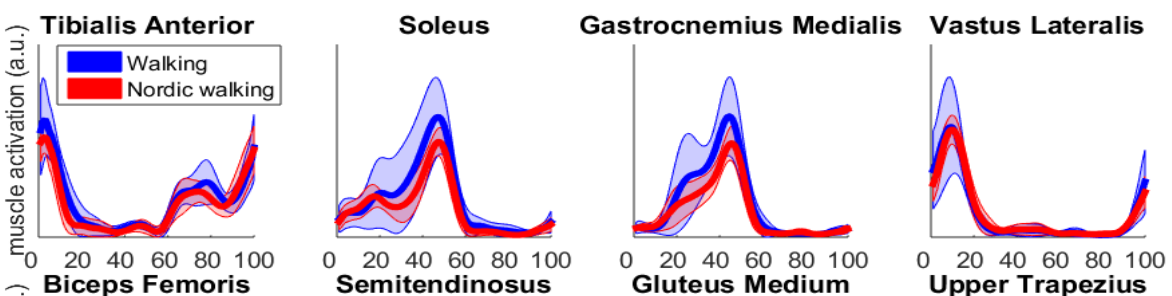

Rectus Femoris

Biceps Femoris

Gluteus Medium

Upper Trapezius
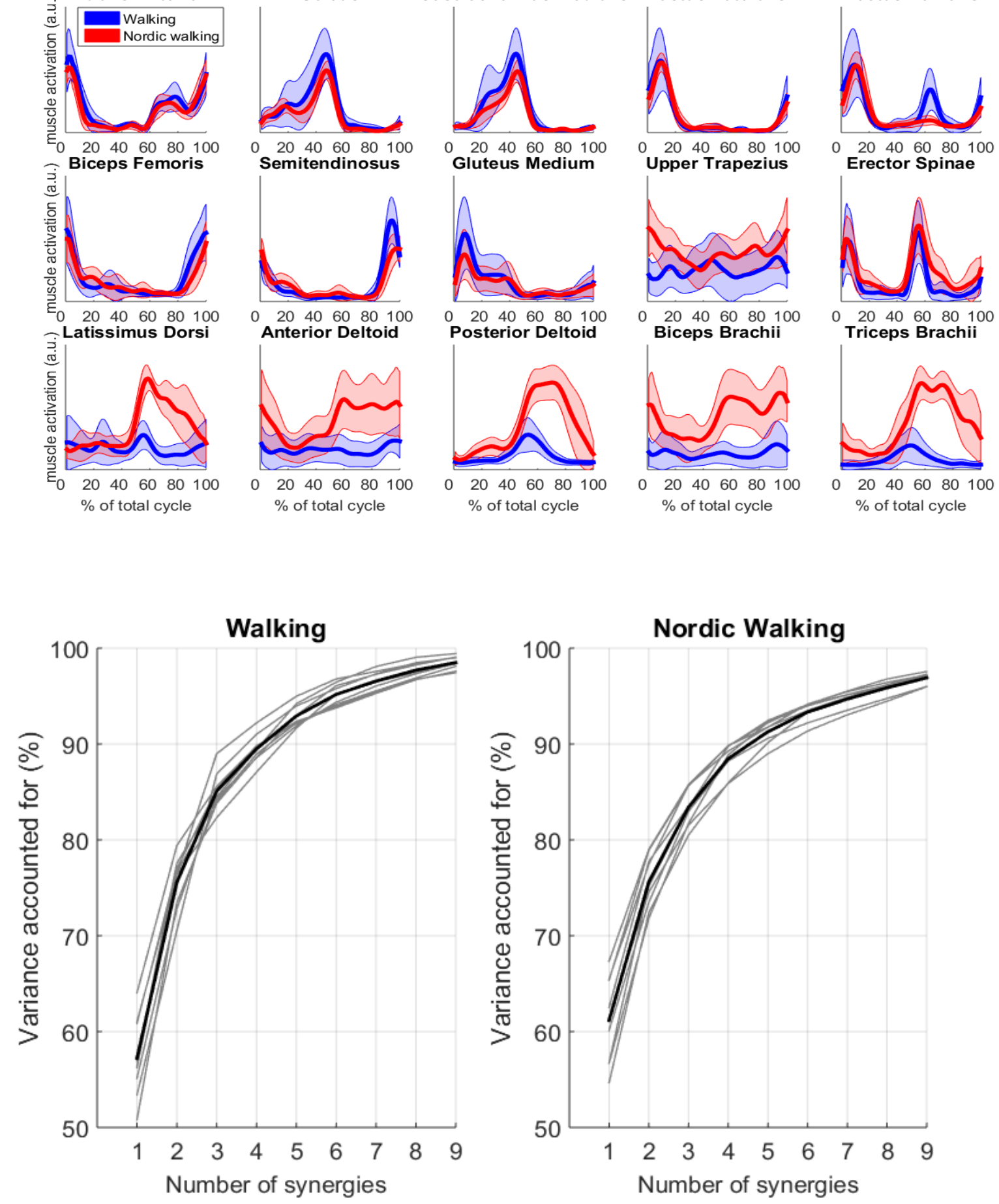

This article is protected by copyright. All rights reserved. 

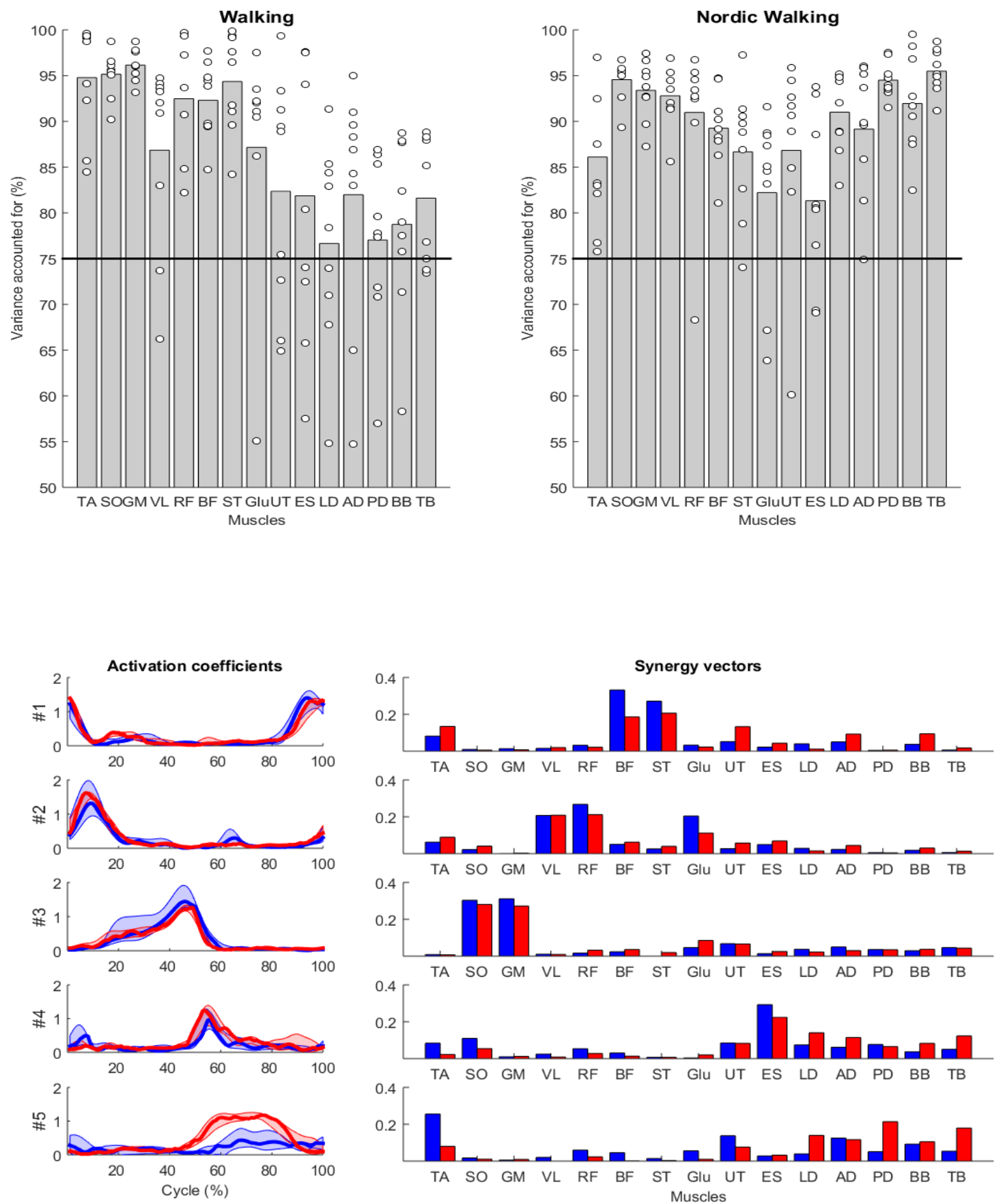

This article is protected by copyright. All rights reserved. 


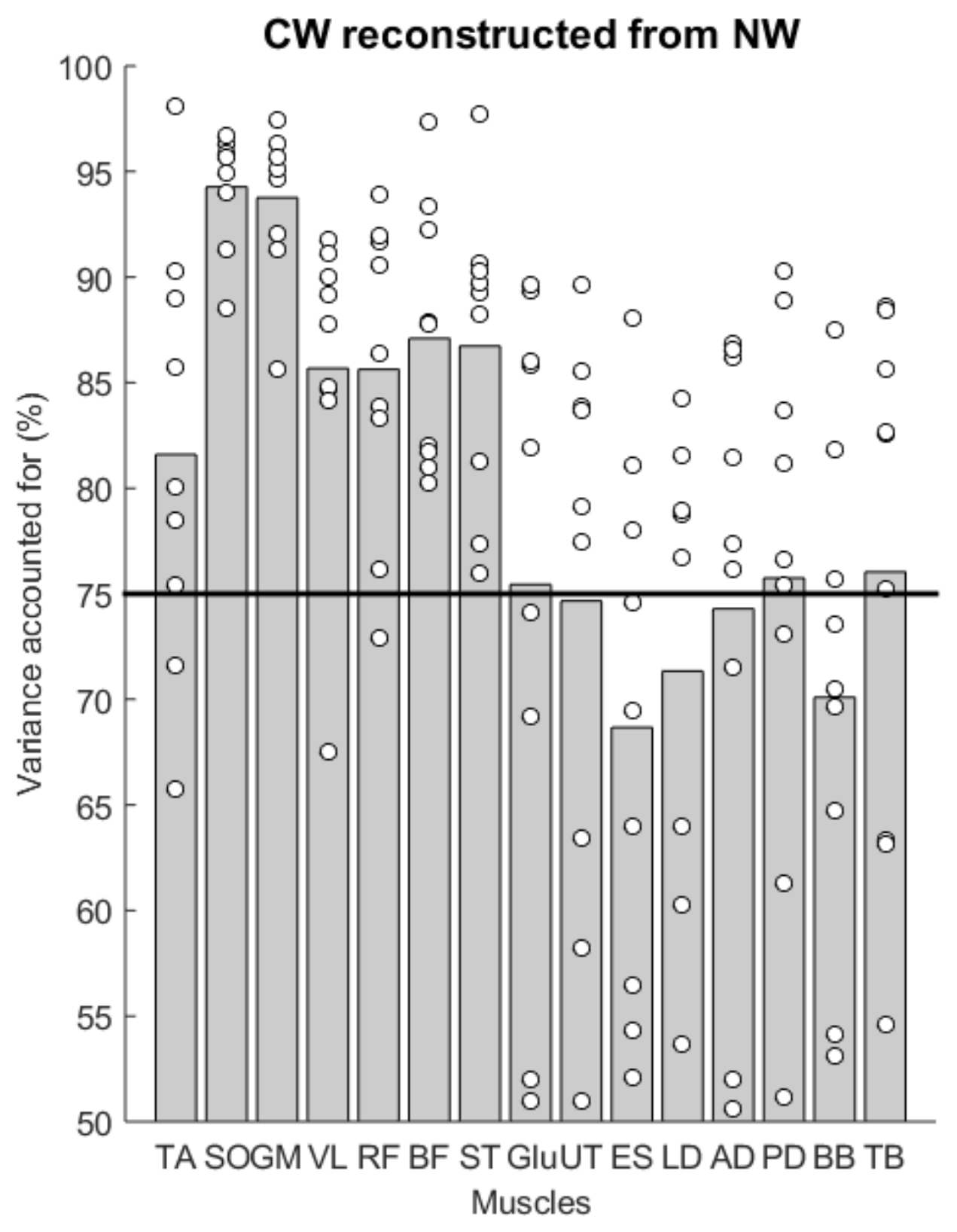

This article is protected by copyright. All rights reserved. 